\title{
Black Leaf Streak Disease in Martinique
}

Present in most areas of banana production, the Black Leaf Streak Disease, commonly called Black Sigatoka, is a banana disease with a higher virulence and broader spectrum of action than the Sigatoka Disease, also called Yellow Sigatoka. A few remaining bastions, including the West Indies, have been spared by this dreaded disease. These bastions are falling one by one.

Detected in January 2010 in St. Lucia, the Black Leaf Streak Disease just made its appearance in Martinique last September, with a very rapid spread throughout the island. The border control measures would not suffice to prevent its entry. They were foiled by an uncontrollable element: the wind.

Already highly sensitized to the risk of disease onset, the crop protection services, CIRAD researchers, growers and agricultural officials have reacted together very quickly to take initial measures to minimize the impact.

We have here an illustrative example of the great advantage in anticipating risks to react as quickly and efficiently as possible when the event happens, and knowing what organization to put in place to cope with it.

It is hoped that, through the implementation of appropriate measures and coordinated and supportive action of professionals, public services and researchers, Martinique can continue this agricultural activity, essential for the economy of the island.

Dr. Jacky Ganry Scientific Director of Fruits

\section{La Maladie des Raies Noires en Martinique}

Présente dans la plupart des zones de production bananière, la Maladie des Raies Noires également appelée cercosporiose noire, constitue une des contraintes phytosanitaires les plus fortes avec une virulence et un spectre d'action beaucoup plus large que la Maladie de Sigatoka, dite cercosporiose jaune. Restaient quelques bastions épargnés par cette maladie tant redoutée, dont faisaient parties les petites Antilles. Ces bastions sont entrain de tomber un par un.

Détectée en janvier 2010 à Sainte-Lucie, la Maladie des Raies Noires vient de faire son apparition en Martinique en septembre dernier, avec une dissémination très rapide sur l'ensemble de l'île. Les mesures de contrôle aux frontières n'auront pas suffi à éviter son entrée. Elles ont été déjouées par un élément non contrôlable : le vent.

Fortement sensibilisés aux risques d'arrivée de la maladie, les services de protection des végétaux, les chercheurs du Cirad, les autorités locales, les produc- teurs et les responsables agricoles ont réagi ensemble très rapidement pour prendre les premières mesures permettant d'en minimiser l'impact.

Nous avons là un exemple illustratif du grand intérêt qu'il y a à anticiper les risques pour réagir le plus rapidement et le plus efficacement possible quand l'événement arrive, et de savoir quelle organisation mettre en place pour $y$ faire face.

Il nous reste à espérer que, grâce à la mise en œuvre des mesures qui s'imposent et à l'action coordonnée et solidaire des professionnels, des services publics et des chercheurs, la Martinique pourra continuer cette activité agricole essentielle pour l'économie de l'île.
Dr. Jacky Ganry Directeur scientifique de Fruits 